\title{
Alternative strategy in crop protection: protease inhibitors from turmeric
}

\begin{abstract}
In an effort to meet the increasing demand for food arising from the growing human population, it is important to ensure food security by maintaining the continual supply of crop products and increase their productions. However, crops plantations are often challenged by the presences of pest insects and pathogens that could inflict diseases or feed on the crop plants and lead to massive losses in the crop productions. While chemical pesticides are commonly employed to control pest insects and pathogens problems, it is often associated with numerous negative side effects and excessive usage would cause lasting detrimental effects to the environments and consumers. Alternatively, crop plants with improved traits were produced through the application of biotechnology techniques to provide phytoprotection against pest insects and pathogens. Genes that encode for natural plant defence products, such as protease inhibitors, are genetically engineered into the crop plants and it is reported to be effectively showing insecticidal and anti-pathogenic properties. For this purpose, it is crucial to constantly discover uncharacterized protease inhibitors from novel sources as candidate for phytoprotection as this helps to overcome the adaptation and resistance buildup by the pest insects and pathogens. Turmeric plant is a well-known herbal plant commonly used as traditional medicine and it acts as a suitable novel source for discovery of protease inhibitors. As turmeric's secondary metabolites are reported to exhibit a wide range of medicinal properties, it could be contributed by protease inhibitors which possessed high anti-pathogenic and inhibitory properties.
\end{abstract}

Keyword: Plant protease inhibitor; Turmeric; Phytoprotection; Genetic engineering; Antipathogenic 